Rechtsmedizin $2010 \cdot 20: 145-146$

DOI 10.1007/s00194-010-0675-3

๑) Springer-Verlag 2010

\author{
M. Parzeller ${ }^{1} \cdot$ R. Dettmeyer ${ }^{2}$ \\ ${ }^{1}$ Institut für Rechtsmedizin, Klinikum der Johann Wolfgang Goethe- \\ Universität Frankfurt am Main, Frankfurt am Main \\ ${ }^{2}$ Institut für Rechtsmedizin, Justus-Liebig-Universität Gießen, Gießen
}

\section{Schutz von Kindern und Jugendlichen vor körperlicher und sexueller Gewalt im normativen Kontext}

Als im Jahr 2009 die ersten Planungen für das Leitthema der rechtlichen Aspekte zur körperlichen Misshandlung, der Vernachlässigung und des sexuellen Missbrauchs von Kindern und Jugendlichen vorgenommen wurden, konnte noch nicht damit gerechnet werden, dass diese Thematik im ersten Quartal 2010 durch die Meldungen über sexuellen Missbrauch und Misshandlung von Kindern in institutionellen Einrichtungen eine zusätzliche Brisanz erfahren würde.

In der politischen und gesellschaftlichen Diskussion wird nun hektisch u. a. über weitere Strafverschärfungen, Änderungen der Verjährungsvorschriften und die Einrichtung von einem oder mehreren runden Tischen debattiert. Schnell entzündet sich ein ministeriales Kompetenzgerangel, welches Ressort (Bundesministerium der Justiz, Bundesministerium für Familie, Senioren, Frauen und Jugend oder Bundesministerin für Bildung und Forschung) die Federführung übernehmen soll. Am 24.03.2010 beschließt das Bundeskabinett die Einrichtung des runden Tisches „Sexueller Kindesmissbrauch in Abhängigkeits- und Machtverhältnissen in privaten und öffentlichen Einrichtungen und im familiären Bereich“, der erstmalig am 23.04.2010 tagen soll. Eine unabhängige Beauftragte in Person der ehemaligen Bundesfamilienministerin Dr. Christine Bergmann soll Opfern und Betroffenen als Anlaufstelle dienen. Zwei verschiedene Arbeitsgruppen des runden Tisches sollen unter Einbeziehung von Vertretern aller gesellschaftlichen Gruppen, von Kinderschutzverbänden, Bera- tungseinrichtungen für Opfer von sexueller Gewalt etc. einerseits Vorschläge zu den Bereichen „Prävention, Intervention, Information" erarbeiten und andererseits zu rechtlichen, rechtspolitischen sowie opferspezifischen Fragen Stellung beziehen $[1,2]$.

Jeder rechtsmedizinisch tätige Arzt kann im Rahmen seiner beruflichen Aufgaben mit Fällen körperlicher und/oder sexueller Gewalt an Kindern und Jugendlichen befasst werden. Als Sachverständiger wird der Rechtsmediziner von den Ermittlungsbehörden, dem Jugendamt, der Heimaufsicht oder einem Gericht zur Klärung des Sachverhalts mit der Untersuchung des Opfers und/oder des Täters beauftragt, aber auch konsiliarisch von Kinderkliniken und niedergelassenen Ärzten um Rat gebeten. Obwohl in der aktuellen und aufgeregten Diskussion der Eindruck entstehen könnte, dass der normative Schutz von Kindern und Jugendlichen vor körperlicher und sexueller Gewalt nicht ausreichen könnte, ist v. a. in den letzten 15 Jahren ein umfangreiches Regelwerk entstanden. Das Themenheft hat zum Ziel, den rechtsmedizinischen Kollegen einen Überblick über einschlägige Gesetzesnormen zum Schutz von Kindern und Jugendlichen vor körperlicher Gewalt und sexuellem Missbrauch zu verschaffen. In den unterschiedlichsten Rechtsgebieten (internationale Normen, Grundgesetz, Strafgesetzbuch, Bürgerliches Gesetzbuch, Sozialgesetzbuch, Verfahrensrechte etc.) liegen einschlägige Normen vor, die verschiedene Institutionen mit Aufgaben und Funktionen zum
Schutz von Kindern und Jugendlichen betrauen. So etabliert sich auf der Grundlage des staatlichen Wächteramts und zahlreicher gesetzlicher Änderungen die Verantwortungsgemeinschaft aus Jugendamt und Familiengericht $[7,8]$. In den rechtlichen Beiträgen, die auf der Analyse mehrerer 100 Entscheidungen der höchstrichterlichen Rechtsprechung der letzten Jahre und der aktuellen Literatur entstanden, werden für die tägliche Arbeit der Rechtmedizin relevante Normen und Sachverhalte erläutert. Dabei werden auch Fehler in den jeweiligen Verfahren angesprochen, die der zeitnahen Wahrheitsfindung abträglich sein können. Rechtliche Aspekte der verschiedenen Rechtsgebiete, die die Arbeit des Sachverständigen betreffen, wurden in die Beiträge einbezogen.

Dramatische Verläufe mit dem Tod von Kindern werden in einer institutsübergreifenden Forschungsarbeit zur tödlichen Vernachlässigung durch Verhungern und Verdursten [9] sowie einer Rechtsprechungsanalyse von Urteilen des Bundesgerichtshofs (BGH) zur Tötung von Neugeborenen und Kindern [10] erörtert. Formen sexueller und körperlicher Gewalt werden anhand einschlägiger Urteile der letzten Jahre in unterschiedlichen Beiträgen dargestellt $[7,8,10,11,12]$. Wichtige Fragen zum Verfahrensrecht und Regelwerke zum Opferschutz werden ebenfalls aufgezeigt [13].

In der Rubrik der zertifizierten Fortbildung wird anhand des Münchhausen-byProxy-Syndroms eine spezielle Form der Kindesmisshandlung erörtert [14]. 
Die Gesetzeslage und die Rechtsprechung entsprechen dem Bearbeitungszeitpunkt der eingereichten Beiträge. Aufgrund der komplexen rechtlichen Materie und in Rechtsprechung und juristischer Literatur strittiger Fragen kann ein Themenheft nur einen knappen Überblick liefern und erhebt nicht den Anspruch auf vollständige Erörterung aller Streitfragen. Die jeweils aktuellen Bundesgesetze können über die Internetseiten des Bundesministeriums der Justiz abgerufen werden [3]. Die in den Beiträgen mit Aktenzeichen angeführten Urteile sind teilweise auf den Internetseiten des jeweiligen Gerichts (z. B. [4]) oder bei entsprechender universitärer Zugangsberechtigung über juristische Internetseiten im Volltext einsehbar $[5,6]$.

Sowohl zur Prävention von körperlicher und sexueller Gewalt an Kindern und Jugendlichen als auch zur Wahrheitsfindung in Ermittlungs- oder Gerichtsverfahren leistet die universitäre Rechtsmedizin einen erheblichen Beitrag. Rechtsmedizinische Studien und Kasuistiken zeigen Formen, Verläufe und Ursachen von Gewalt gegen Kinder und Jugendliche. Die Rechtsmedizin trägt durch die Sensibilisierung angehender Kollegen bereits in der Lehre sowie durch ärztliche und sonstige Fortbildung, z. B. für Polizeibeamte und Mitarbeiter von Jugendämtern, dazu bei, Formen körperlicher und sexueller Gewalt frühzeitig zu erkennen. Dadurch können die zuständigen Institutionen Hilfs- und Schutzmaßnahmen vor weiteren Verletzungen der körperlichen Integrität des Opfers ergreifen. Gleichwohl ist zu bedenken, dass sich nicht jeder Sachverhalt als eindeutiger Fall einer Kindesmisshandlung oder des sexuellen Missbrauchs darstellt. Als objektive, neutrale und sachverständige Institution ist die universitäre Rechtsmedizin in einer interdisziplinären und institutionellen Zusammenarbeit ein kompetenter Ansprechpartner zum Schutz des Kindes, zur Wahrung der Elternrechte und bei der Wahrheitsfindung. Diese verantwortungsvolle Aufgabe muss aber mehr als bisher vonseiten des Staates durch entsprechende finanzielle Mittel und die Bereitstellung aus- reichender personeller Ressourcen unterstützt und gefördert werden.

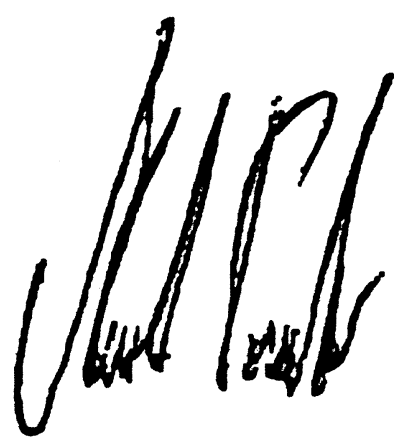

Markus Parzeller (Frankfurt)
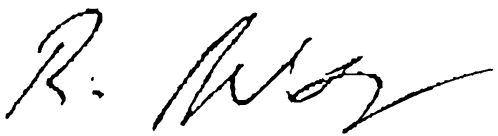

Reinhard Dettmeyer (Gießen)

\section{Korrespondenzadressen}

PD Dr. med. Dr. med. habil. Ass.

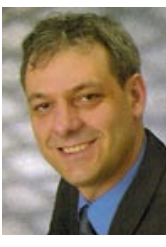

jur. M. Parzeller

Institut für Rechtsmedizin, Klinikum der Johann Wolfgang Goethe-Universität Frankfurt am Main Kennedyallee 104, 60596 Frankfurt am Main parzeller@em.uni-frankfurt.de

Prof. Dr. med. Dr. jur. R. Dettmeyer

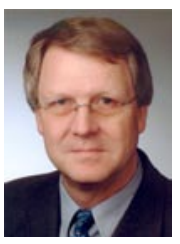
Institut für Rechtsmedizin, Justus-Liebig-Universität Gießen, Frankfurter Str. 58, 35392 Gießen Reinhard.Dettmeyer@forens. med.uni-giessen.de

Interessenkonflikt. Der korrespondierende Autor gibt an, dass kein Interessenkonflikt besteht.

\section{Literatur und Quellennachweis}

1. www.tagesschau.de/inland/missbrauchsskandal110.html v. 24.03.2010

2. www.bmfsfj.de/BMFSFJ/kinder-undjugend,did=134436.html v. 24.03.2010

3. www.gesetze-im-internet.de

4. www.bundesgerichtshof.de

5. http://beck-online.beck.de/default.aspx

6. http://www.juris.de/jportal/Zugang.jsp\#
7. Parzeller M, Dettmeyer R, Bratzke H et al (2010) Schutz von Kindern vor Gewalt - Rechtliche Vorgaben in der UN-Kinderrechtskonvention und im Grundgesetz. Rechtsmedizin, doi 10.1007/s00194010-0670-8

8. Parzeller M, Dettmeyer R, Bratzke $H$, Zedler B (2010) Körperliche Misshandlung und sexueller Missbrauch von Kindern und Jugendlichen - Rechtliche Vorgaben im Zivil- und Sozialrecht. Rechtsmedizin, doi 10.1007/s00194-010-0672-6.

9. Riße M, Rummel J, Tsokos M et al (2010) Verhungern und Verdursten. Rechtsmedizin, doi 10.1007/ s00194-010-0674-4.

10. Parzeller M, Zedler B, Bratzke H, Dettmeyer R (2010) Tödliche Gewalt gegen Kinder - Strafrechtliche Aspekte anhand einschlägiger höchstrichterlicher Rechtsprechung. Rechtsmedizin, doi 10.1007/s00194-010-0655-7.

11. Parzeller M, Dettmeyer R, Flaig B et al (2010) Straftaten gegen die sexuelle Selbstbestimmung und gegen die persönliche Freiheit von Kindern und Jugendlichen. Rechtsmedizin, doi 10.1007/ s00194-010-0671-7

12. Parzeller M, Zedler B, Bratzke $H$, Dettmeyer $R$ (2010) Körperverletzung, Aussetzung und Verletzung der Fürsorgepflicht gegenüber Kindern Strafrechtliche Aspekte anhand höchstrichterlicher Rechtsprechung. Rechtsmedizin, doi 10.1007/ s00194-010-0665-5.

13. Dettmeyer R, Zedler B, Bratzke H et al (2010) Ausgewählte verfahrensrechtliche Aspekte bei Gewalt gegen Minderjährige. Rechtsmedizin, doi 10.1007/ s00194-010-0673-5.

14. Noeker M, Mußhoff F, Franke I, Madea B (2010) Münchhausen-by-Proxy-Syndrom. Rechtsmedizin 20, doi 10.1007/s00194-009-0642-z. 